\title{
An Approach to Adaptive Enhancement of Diagnostic X-Ray Images
}

\author{
Hakan Öktem \\ Institute of Signal Processing, Tampere University of Technology, P.O. Box 553, 33101 Tampere, Finland \\ Email: oktem@cs.tut.fi \\ Karen Egiazarian \\ Institute of Signal Processing, Tampere University of Technology, P.O. Box 553, 33101 Tampere, Finland \\ Email:karen@cs.tut.fi
}

\author{
Jarkko Niittylahti \\ Atostek Ltd., Hermiankatu 8D, FIN-33720 Tampere, Finland \\ Email: jarkko.niittylahti@atostek.com
}

Juha Lemmetti

Atostek Ltd., Hermiankatu 8D, FIN-33720 Tampere, Finland

Email: juha.lemmetti@atostek.com

Received 31 January 2002 and in revised form 3 October 2002

\begin{abstract}
Digital radiography is a popular diagnostic imaging method. Denoising and enhancement have an important potential in obtaining as much easily interpretable diagnostic information as possible with reasonable absorbed doses of ionising radiation. Due to the increasing usage of high resolution and high precision images with a limited number of human experts, the computational efficiency of the denoising and enhancement becomes important. In this paper, a local adaptive image enhancement and simultaneous denoising algorithm for fulfilling the requirements of digital X-ray image enhancement is introduced. The algorithm is based on modification of the wavelet transform coefficients by a pointwise nonlinear transformation and reconstructing the enhanced image from the modified wavelet transform coefficients. The implementation of algorithm in software is simple, quick, and universal.
\end{abstract}

Keywords and phrases: image enhancement, X-ray images, wavelet shrinkage.

\section{INTRODUCTION}

Typically, digital X-ray images are corrupted by additive noise relatively higher with respect to conventional X-ray films. Higher SNR is possible at cost of higher absorbed doses of ionising radiation. Furthermore, image enhancement algorithms generally amplify the noise $[1,2,3,4]$. Therefore, higher denoising performance is important in obtaining images with high visual quality using relatively lower doses of ionising radiation. The most important part of the corrupting noise is the Gaussian noise whose variance may vary with the signal level (due to sensor nonlinearity) and spatially depending on the instrumentation [2]. The visibility of some structures in medical X-ray images, especially the details that may be conveying diagnostic information, may have a vital role in providing sufficient visual information for the clinician. The visibility of relatively smaller and nonsignificant details may be extremely important, especially in early diagnosis of cancer. Another important aspect here is the computational efficiency. The algorithm should be executed in a reasonable time since the number of human experts is limited and the workloads of radiological units are increasing especially due to the screening policies. The accuracy and resolution of X-ray images are also increasing, thus requiring more computations to be performed.

Among different adaptive image enhancement methods, adaptive unsharp masking, adaptive neighbourhood filtering and enhancement, adaptive contrast enhancement, and various adaptive filtering approaches by directional wavelet transform (WT) $[5,6,7,8]$ can be mentioned. However, most of these methods involve a priori information about the image $[3,5]$. Some images, in particular, thorax images, include information on many different tissues with different $\mathrm{X}$-ray transmittance, and even normal variations in the data 
may affect the performance and reliability of the algorithm. In this paper, we propose an enhancement algorithm which does not require any a priori anatomical information.

We introduce the problem in Section 2, the image enhancement algorithm in Section 3, simulation results in Section 4, and, finally, we conclude in Section 5.

\section{DESCRIPTION OF THE PROBLEM}

After discussing the potential effects of image denoising and enhancement for the digital radiographic images, we can proceed by discussing the specific needs of enhancing the diagnostic X-ray images. There are three important issues to be considered.

(1) X-ray images (especially thorax images) include different regions containing details. Both sharp and soft transitions between the regions and details may exist in all visual spans. When all details are enhanced to the same extent, the relatively significant details cover most of the visual span and prevent the visibility of relatively less significant details. This is illustrated in Figure 1.

(2) Since X-ray images are used for diagnostic purpose, the image enhancement must not cause misleading information, making a structure looking more or less significant than it is must be avoided.

(3) Data loss is not desirable in diagnostic images. Therefore, the noise attenuation procedure must not remove any visual information.

Another problem with X-ray (especially thorax) images is the risk of incorporating a priori information about the visual structures of the image for enhancement and denoising purpose. Unlike the common images, X-ray images are rendered volume data and the transitions between the same structures may be smooth or sharp depending on the angle. The images generally belong to known anatomic regions but the visual features corresponding to anatomical structures are not unique every time. The varying transitions for the same object are illustrated in Figure 2 [4].

The WT is a transform decomposing an image into approximations and details at different resolution levels $[8,9$, 10]. Since we can express the original image as a combination of its approximations and different levels of details, we can build a simultaneous denoising and enhancement algorithm in WT domain according to the requirements listed above in this section.

\section{ALGORITHM}

\subsection{Wavelet transform}

The WT of a signal $f(x)$ at a scale $s$ and shift $t$ is defined as

$$
W_{s, t} f(x)=\left\langle f(x) \cdot \Psi_{s, t}(x)\right\rangle=\frac{1}{\sqrt{s}} \int_{-\infty}^{\infty} f(t) \Psi\left(\frac{x-t}{s}\right) d x,
$$

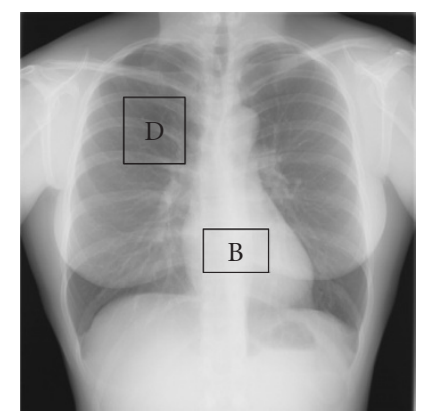

(a)

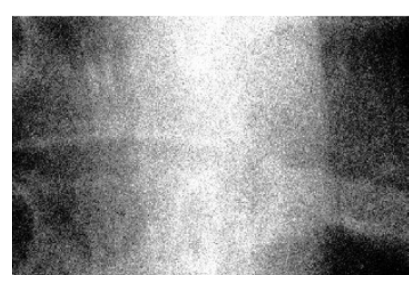

(c)

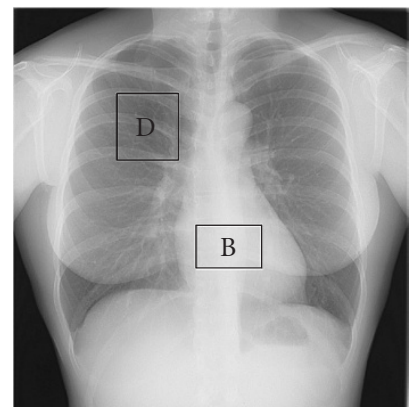

(d)
FIGURE 1: (a) An original thorax image whose histogram was adjusted using commercial software. "D" is a portion of soft tissue region and " $\mathrm{B}$ " is a bone region. (b) The region " $\mathrm{D}$ " of Figure 1a after edge enhancement applied within the region. (c) The region " $\mathrm{B}$ " of Figure 1a after histogram equalisation applied within the region. (d) The sharpened version of the original image. As we can see from the image, the most significant details in the original image, which are already visible, are enhanced. However, nonsignificant details, like those present in the region "B", such as the bottom parts of the image and so forth, are not visible anymore. This is mainly due to the limits of the visual span. Whatever we do to the image, we can always represent the brightest pixel with the maximum and darkest pixel with the minimum brightness of the screen. Furthermore, this sharpened image may even cause misleading distortions since some vessels look more significant than the bones due to the highfrequency content of the relatively thin structures (this distortion is very clear especially around the 4th rib from the bottom). One alternative to improve the visibility will be to apply stronger enhancement to the relatively nonsignificant details, and relatively weaker enhancement for already visible details is an alternative for improving the visual information and solving the first problem. This brings the necessity of adaptive enhancement.

where $\Psi(x)$ is the mother wavelet and $\Psi_{s, t}(x)$ is the scaled (stretched) and shifted version of the mother wavelet $[9,10$, $11]$.

When the shift $t$ is sampled at integers and the scale $s$ is sampled at integer powers of two, the shifted $\Psi(x-t)$ and scaled $\Psi(x / s)$ versions of a main wavelet function $\Psi(x)$ form a basis. The basis functions are denoted by $\Psi_{s, t}(x)$ [12]. 


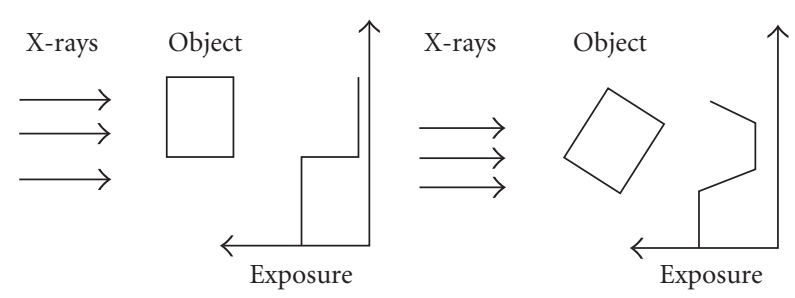

FIgURe 2: An illustration of the varying transitions in the X-ray images.

Let

$$
C(j, k)=\left\langle f(t) \cdot \Psi_{j, k}(t)\right\rangle=\sum_{n \in Z} x(n) g_{i, k}(n)
$$

be the discrete wavelet transform (DWT) coefficients of signal $f(t)$ and let $\Psi_{j, k}(t)$ be an orthogonal wavelet function. Reconstruction of the signal from its WT coefficients at different scales gives the details $D$ (high-frequency information) and approximations $A$ (low-frequency information) of the signal at level $j$ defined as

$$
\begin{aligned}
D_{j}(t) & =\sum_{k \in Z} C(j, k) \Psi_{j, k}(t), \\
f(t) & =\sum_{k \in Z} D_{j}, \\
A_{J} & =\sum_{j>J} D_{j}, \\
A_{J-1} & =A_{J}+D_{J} .
\end{aligned}
$$

Iterated two-channel filter banks can be used to perform the wavelet decomposition (see Figure 3, where LPF and HPF are analysis lowpass and highpass filters, resp.).

The downsampled outputs of the highpass filter are detail coefficients, and the downsampled outputs of the lowpass filter are approximation coefficients. The detail and approximation coefficients provide an exact representation of the signal, thus no information is lost during downsampling. Decomposing the approximation coefficients perform a further level of the detail and approximation coefficients $[10,11$, $13]$.

The reconstruction process is done by inverse iterative two-channel filter bank, consisting of upsampling from each channel, performing a synthesis lowpass and highpass filtering, and summing up the results from both channels [11] (see Figure 4 with $\alpha=1$ and $g(x)=x$, for one stage of reconstruction).

Nonlinear modification of wavelet detail coefficients is an efficient way to perform an adaptive image enhancement. Furthermore, eliminating the detail coefficients whose magnitude lies under a threshold is an efficient denoising technique, called wavelet shrinkage [14].

\subsection{Description of the algorithm for simultaneous $X$-ray image denoising and enhancement}

The algorithm is partially graphically illustrated in Figure 4 . First, the wavelet decomposition is performed. Then, the transform coefficients are modified by a special pointwise function followed by the inverse WT.

The modification of WT coefficients and computation of the enhanced and denoised images from the modified transform coefficients can be described in the following steps. ${ }^{1}$

(1) The detail coefficients with absolute values under the threshold $t$ are attenuated by an exponentially increasing point transformation normalized between 0 and $t$. The coefficients with absolute values higher than $t$ are not modified, that is,

$$
x_{N}(i, j)= \begin{cases}D_{N}(i, j), & \text { if }\left|D_{N}(i, j)\right| \geq t, \\ t \operatorname{sgn} D_{N}(i, j) \frac{e^{D_{N}(i, j) / k}-1}{e^{t / k}-1}, & \text { otherwise, }\end{cases}
$$

where $\operatorname{sgn}(\cdot)$ is the sign function. This operation is used for noise attenuation instead of hard or soft thresholding used in wavelet shrinkage $[7,8]$. The reason for this is following. The hard thresholding may introduce some artefacts while soft thresholding causes attenuation of relatively nonsignificant details conflicting with the enhancement requirements. The coefficients corresponding to low SNR are attenuated instead of totally removing them. The operation in (4) is invertible, no information is lost, and the original image can be recovered. This is important especially for diagnostic images. Here, $t$ and $k$ are user specified tuneable adjustment parameters. The "optimum" threshold $t$ for identically distributed white Gaussian noise is given by $\sigma \sqrt{2 \log m}$, where $\sigma$ is the noise standard deviation and $m$ is the number of transform coefficients [14]. However, for diagnostic images, assistance of a human expert is needed.

(2) After noise attenuation, the coefficients are modified by a point transformation $b(i)=f(a(i))$ (where $a(i)$ and $b(i)$ are arbitrary variables), such that details with lower magnitude are enhanced more than the details having higher magnitude, but do not exceed them. In this way, the following two properties are satisfied:

(i) if $|a(i)|>|a(j)|$, then $|b(i)|>|b(j)|$,

that is, if a local detail is more significant than another local detail at the same resolution in the original image, it is also more significant in the enhanced image; ${ }^{2}$

\footnotetext{
${ }^{1}$ Since the operations in steps (1), (2), and (3) are only pointwise modifications, the first three steps can be performed by a single pointwise modification, shown as $g(\cdot)$ in Figure 4.

${ }^{2}$ Clinicians observe the following problem with an image enhancement. It is known that malignant tumors increase blood flow to themselves. In enhanced image, some of the vessels may look more significant than they really are which may lead to a wrong conclusion. We presume that preserving the order of the contrasts of structures at each resolution, which can be approximated with wavelet detail coefficients, will help to handle this problem.
} 


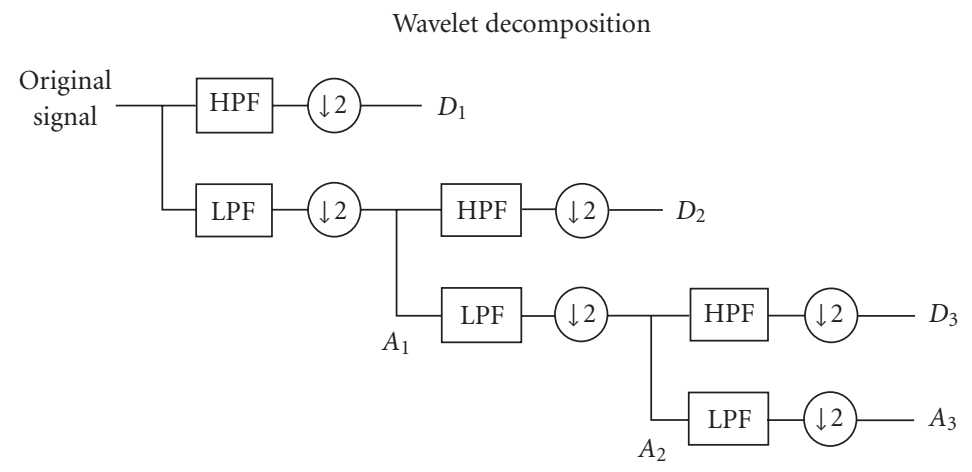

FIGURE 3: The illustration of wavelet decomposition by filter banks.

Decomposition

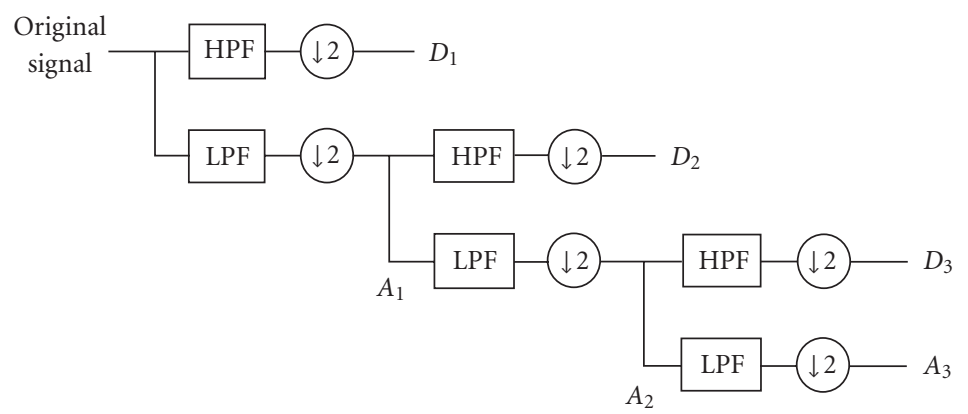

Reconstruction of enhanced and denoised image

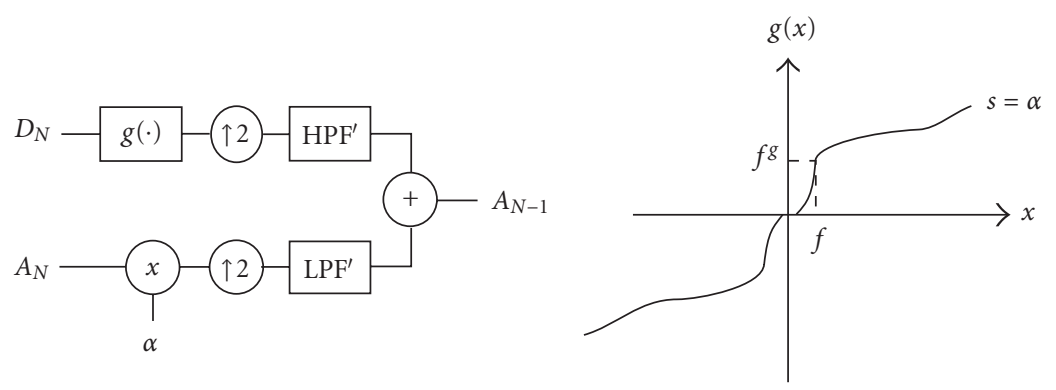

FIgURE 4: The graphical illustration of the algorithm.

(ii) if $|a(i)|>|a(j)|$, then $|\partial a / \partial b|_{a=a(i)}<|\partial a / \partial b|_{a=a(j)}$,

which provides a stronger enhancement for relatively less significant details.

We have used a root operation

$$
y(i, j)=\operatorname{sgn}(x(i, j))|x(i, j)|^{\gamma}, \quad 0<\gamma<1,
$$

as a typical example satisfying the desired properties. Here, $x(i, j)$ is the output of step (1) (detail coefficients after noise attenuation step), $\gamma$ is a tuneable parameter of the algorithm controlling the enhancement level. Small $\gamma$ provides higher enhancement and the enhanced image converges to the original image when $\gamma$ approaches 1 .
(3) The enhanced detail coefficients are prevented to attenuate more than the approximations, that is,

$$
y^{\prime}(i, j)=\operatorname{sgn}(y(i, j)) \max (|y(i, j)|, \alpha|x(i, j)|),
$$

where $y(i, j)$ is the output of step (2) and $x(i, j)$ is the output of step (1) (before the enhancement is applied) and $\alpha$ is the coefficient multiplied by the approximation coefficients.

(4) The approximation coefficients are attenuated according to

$$
A^{\prime}(i, j)=\alpha A(i, j), \quad 0<\alpha<1,
$$

in order to decrease the contribution of low frequencies. 
Here, we took $\alpha$ as another tuneable parameter, specified by a user.

(5) Each lower level of approximation coefficients is computed by using the modified detail and approximation coefficients of the previous level of reconstruction. Since this low frequency attenuation is applied at each step, $N$ th level of approximations are attenuated by $\alpha^{N}$.

(6) The reconstruction continues until the final enhanced image is computed.

Due to downsampling, a WT is not translation invariant and the algorithms based on nonlinear modification of the WT coefficients introduce some artefacts. In [15], translation invariant denoising scheme is presented, where the wavelet denoising is performed for all possible translations of the signal and the results are averaged (cycle-spinning). As an alternative, a partial cycle spinning can be performed by arbitrarily selected (not necessarily all) shifts of the signal.

\subsection{Computational complexity analysis}

The filter bank implementation of the wavelet decomposition is a computationally efficient way to obtain the multiresolution representation of the image. The modification function is a combination of pointwise modifications with the maximum complexity of finding from a lookup table.

The computation consists of wavelet decomposition, pointwise nonlinear modification, and reconstruction. The decomposition involves horizontal and vertical filtering with downsampling by two. Both of these tasks take $O\left(l_{f} \cdot M\right)$ operations, where $l_{f}$ is the length of the filter and $M$ is the amount of pixels in the image. The complexity of the nonlinear modification is also directly proportional to the amount of pixels in the image. The reconstruction's computational complexity is equal to the decomposition's complexity $[16,17,18]$.

Thus, the combined complexity is $O\left(l_{f} \cdot M\right)$. The depth of the decomposition does not affect it because the decomposition of level $N$ will take a quarter of the complexity of the decomposition of level $N-1$.

The algorithm was implemented using a typical PC workstation and the C-programming language. The enhancement of one translation for $2000 \times 2000,16$ bit X-ray images took less than 10 seconds to run using a $500-\mathrm{MHz}$ Pentium III computer. When the enhancement is run on a modern 1.7-GHz Pentium IV computer, the time needed for it decreases to less than 3 seconds. The use of dualprocessor workstation reduced the enhancement time by approximately $40 \%$. Because the algorithm is implemented on a general-purpose workstation, the performance can be expected to increase in time with no additional efforts $[16,17$, 18]. The implemented algorithm is very convenient in use due to its fast execution. The commercial software used previously for this application required execution time of $60 \mathrm{sec}-$ onds.

\section{RESULTS}

For testing purpose $2000 \times 2000,12$-bit X-ray images were used, namely, 15 frontal, four sagittal thorax images, and

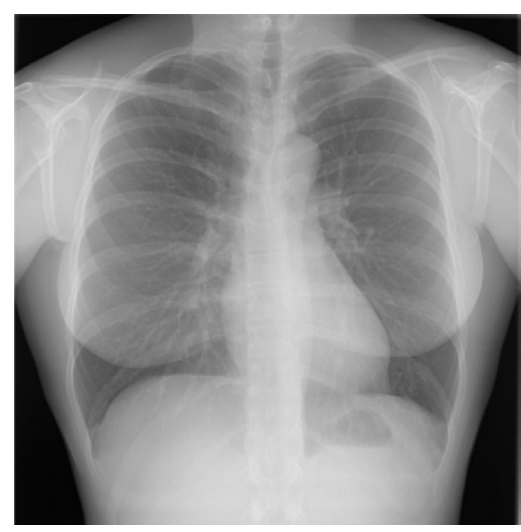

FIgURE 5: The enhanced version (histogram was adjusted by using a commercial software) of the original image in Figure 1. This image was obtained by 8 -level wavelet decomposition using a symlet 8 -filter bank. The parameters are $\gamma=0.92, t=2 \hat{\sigma}, k=0.1$, and $\alpha=0.92$, where $\hat{\sigma}$ is the estimate of the noise standard deviation computed by $\sqrt{2}$ times the median of the coarsest level of detail coefficients $\left(D_{1}\right) .8$ arbitrarily selected shift variants of the enhanced image were averaged for additional suppression of artefacts.

one-hand and one-ankle images. ${ }^{3}$ In our study, evaluation was a part of progress of the research. Test images, denoised and enhanced by various known algorithms (such as unsharp masking [19], highpass filtering [19], histogram modification [19], root filtering [19], classical wavelet shrinkage [9], etc.) were sent to experts from the radiology departments of various hospitals including Helsinki and Tampere University hospitals for their evaluation. They have listed the problems related with these algorithms. Opinions of radiologists were acquired (by support of a provider of X-ray imaging systems) at various steps of the algorithm development and the final algorithm was obtained by confirmation of solution of the reported problems. It should be noted that the algorithm introduced in this work was the only one among various alternatives which was approved by the experts. Two main advantages of the images enhanced using new algorithm are the following. First, both bone details (like spine in the thorax images) and soft tissue details (like the vessels in the thorax images) become visible within the same image. Second, artefacts and deviations discussed in footnote 2 were not noticeable. The enhanced version of the original image in Figure 1 is shown in Figure 5 and the same image with relatively higher rates of enhancement is shown in Figure 6. The algorithm is universal; it does not need any a priori information on the anatomical features.

When the enhancement is performed by a linear filter (like in Figures 1, 2, 3, 4), 2-dimensional convolution is applied requiring $O\left(s_{f} \cdot s_{f} \cdot M\right)$, where $s_{f}$ is the length of the sharpening filter. Furthermore, if the same frequency

\footnotetext{
${ }^{3}$ Courtesy of Imix Ltd., Tampere, Finland.
} 


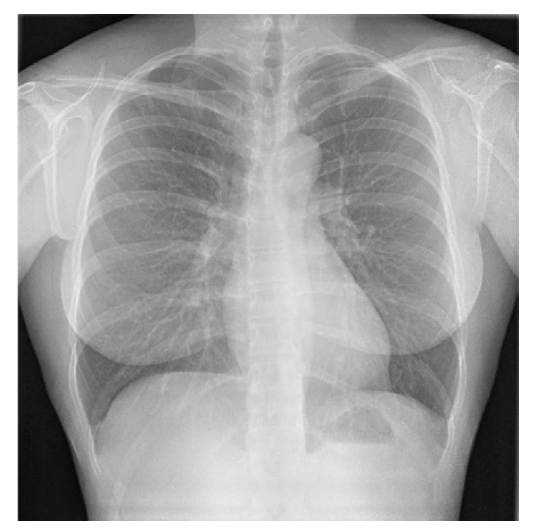

FIGURE 6: The enhanced version (histogram was adjusted by using a commercial software) of the original image in Figure 1 with sharper enhancement parameters with respect to the image in Figure 5. This image is obtained by the same decomposition scheme as in Figure 5. The parameters are $\gamma=0.85, t=2, k=0.1$, and $\alpha=0.85 .8$ arbitrarily selected shift variants of the enhanced image were averaged to suppress artefacts. Such kind of sharply enhanced images are generally not preferred for clinical use. However, even in sharply enhanced images the problems shown in Figure 1 are not observed.

resolution is performed by a sharpening filter, its length $s_{f}$ needs to be $2^{N} \cdot l_{f}$ since the equivalent support of a wavelet filter is doubled in each step of decomposition, due to downsampling.

\section{CONCLUSIONS}

This work aims to improve the visually recognizable information in the diagnostic X-ray images. Algorithm increases the visibility of relatively nonsignificant details without distorting the image and within a reasonable execution time. This is particularly important when the screening is considered. Because the structures due to cancer are progressing in time, recognition of corresponding structures as early as possible has a direct relation with the survival chance of the patient. Improved representation of the diagnostic Xray images will help a human expert to perform an early diagnosis.

\section{ACKNOWLEDGMENTS}

We wish to thank Ms. Mari Lehtimäki for allowing her X-ray film being used for research and publication. Furthermore, we are grateful to Ms. Mari Lehtimäki and Mr. Vesa Varjonen for their fruitful cooperation in the development of the algorithm, supplying the test images and providing feedback on the processed images.

\section{REFERENCES}

[1] T. Aach, U. Schiebel, and G. Spekowius, "Digital image acquisition and processing in medical X-ray imaging," Journal of Electronic Imaging, vol. 8, no. 1, pp. 7-22, 1999.
[2] K. K. Shung, M. B. Smith, and B. M. W. Tsui, Principles of Medical Imaging, Academic Press, San Diego, Calif, USA, 1992.

[3] T. Ozanian, R. Phillips, and A. Mosquera, "An algorithm for enhancement of noisy X-ray images," in 18th Annual International Conference on the IEEE Engineering in Medicine and Biology Society, Amsterdam, The Netherlands, OctoberNovember 1996.

[4] H. Öktem, K. Egiazarian, J. Niittylahti, J. Lemmetti, and J. Latvala, "A wavelet based algorithm for simultaneous X-ray image de-noising and enhancement," in Proc. 2nd International Conference on Information, Communications \& Signal Processing (ICICS '99), Singapore, December 1999.

[5] M. J. Carreira, D. Cabello, A. Mosquera, M. G. Penedo, and I. Facio, "Chest X-ray image enhancement by adaptive processing," in Proc. Annual International Conference on the IEEE Engineering in Medicine and Biology Society, vol. 13, Orlando, Fla, USA, 1991.

[6] S. Guillion, P. Baylou, M. Najim, and N. Keskes, "Adaptive non-linear filters for 2D and 3D image enhancement," Signal Processing, vol. 67, pp. 237-254, 1998.

[7] L. Li, W. Qian, and L. P. Clarke, "X-ray medical image processing using directional wavelet transform," in Proc. IEEE International Conference on Acoustics, Speech, and Signal Processing, pp. 2251-2554, Atlanta, Ga, USA, May 1996.

[8] Z. Hua and M.-N. Chong, "A wavelet de-noising approach for removing background noise in medical images," in Proc. International Conference on Information, Communications \& Signal Processing (ICICS '97), vol. 2D28, pp. 980-983, Singapore, 1997.

[9] S. Mallat, A Wavelet Tour of Signal Processing, Academic Press, San Diego, Calif, USA, 1998.

[10] I. Daubechies, "The wavelet transform: a method of time frequency localization," in Advances in Spectral Analysis, Prentice Hall, Englewood Cliffs, NJ, USA, 1990.

[11] M. Vetterli and Y. Kovacevic, Wavelets and Subband Coding, Prentice Hall, Englewood Cliffs, NJ, USA, 1995.

[12] S. C. Pei and M. H. Yeb, "An introduction to discrete finite frames," IEEE Signal Processing Magazine, vol. 14, no. 6, pp. 84-96, November 1997.

[13] H. S. Malvar, Signal Processing with Lapped Transforms, Artech House, Boston, Mass, USA, 1992.

[14] D. L. Donoho and I. M. Johnstone, "Adapting to unknown smoothness via wavelet shrinkage," Journal American Statistical Association, vol. 90, no. 432, pp. 1200-1224, 1995.

[15] R. R. Coifman and D. L. Donoho, "Translation invariant denoising," in Wavelets and Statistics, A. Antoniadis and G. Oppenheim, Eds., vol. 103 of Lecture Notes in Statistics, pp. 125150, Springer-Verlag, New York, NY, USA, 1995.

[16] J. Niittylahti, J. Lemmetti, and J. Helovuo, "On implementing signal processing algorithms on PC," Microprocessors and Microsystems, vol. 26, no. 4, pp. 173-179, 2002.

[17] J. Lemmetti, J. Latvala, K. Öktem, H. Egiazarian, and J. Niittylahti, "Implementing wavelet transforms for X-ray image enhancements using general purpose processors," in Proc. IEEE Nordic Signal Processing Symposium (NORSIG'2000), Kolmården, Sweden, June 2000.

[18] J. Niittylahti and J. Lemmetti, "Implementation of waveletbased algorithms on general purpose processors," in Proc. International Workshop on Spectral Methods and Multirate Signal Processing, Pula, Croatia, June 2001.

[19] A. K. Jain, Fundamentals of Digital Image Processing, Prentice Hall, Englewood Cliffs, NJ, USA, 1989. 
Hakan Öktem was born in Urfa, Turkey, in 1967. He received the B.S. degree in electrical engineering from Middle East Technical University, Ankara, Turkey in 1990, and the M.S. degree in electrical engineering from Tampere University of Technology, Tampere, Finland in 1998. He is currently working at Tampere University of Technology, Institute of Signal Processing and studying for doctoral degree in infor-

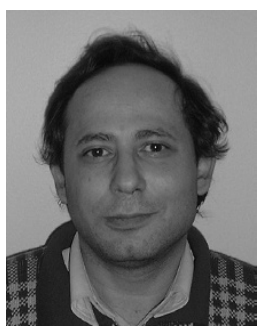
mation technology at Tampere University of Technology. His research interest concern signal and image denoising, image enhancement, transforms, and bioinformatics.

Karen Egiazarian was born in Yerevan, Armenia, in 1959. He received the M.S. degree in mathematics from Yerevan State University in 1981, and the Ph.D. degree in physics and mathematics from M.V. Lomonosov Moscow State University in 1986. In 1994, he was awarded the degree of Doctor in technology by Tampere University of Technology, Finland. He has been a Senior Researcher at the Department of Digital Signal

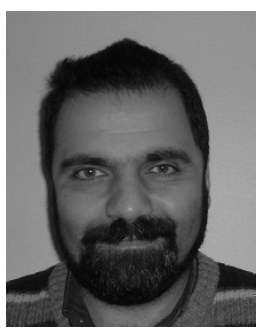
Processing at the Institute of Information Problems and Automation, National Academy of Sciences of Armenia. He is currently a Professor at the Institute of Signal Processing, Tampere University of Technology. His research interests are in the areas of applied mathematics, digital logic, signal and image processing. He has published more than 200 papers in these areas and is the coauthor (with S. Agaian and J. Astola) of Binary Polynomial Transforms and Nonlinear Digital Filters, published by Marcel Dekker, in 1995. Also, he coauthered three book chapters.

Jarkko Niittylahti was born in Orivesi, Finland, in 1962. He received the M.S., Lic.Tech, and Dr.Tech degrees from Tampere University of Technology (TUT) in 1988, 1992, and 1995, respectively. From 1987 to 1992, he was a Researcher at TUT. In 1992-1993, he was a Researcher at CERN in Geneva, Switzerland. In 19931995, he was with Nokia Consumer Elec-

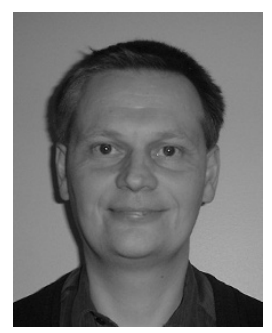
tronics, Bochum, Germany, and in 19951997 with Nokia Research Center, Tampere, Finland. In 1997-2000, he was a Professor at Signal Processing Laboratory, TUT, and in 2000-2002, at Institute of Digital and Computer Systems, TUT. Currently, he is a Docent of Digital Techniques at TUT and the Managing Director of Staselog Ltd. He is also a cofounder and President of Atostek Ltd. He is interested in designing digital systems and architectures.

Juha Lemmetti was born in 1975 in Tampere, Finland. He graduated from digital and computer systems in 2000 at the Tampere University of Technology, Finland. He is currently working at Atostek Ltd. as a Chief of Software Design. He is also a doctoral student in the Institute of Software Systems at the Department of Information Technology, TUT. His main interests concern fast implementations of signal processing algorithms including real-time image processing and video compression.

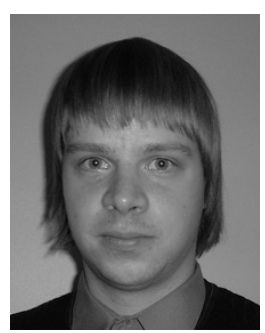$$
\text { compression. }
$$ 